\title{
Un percorso di peer education nella scuola superiore incentrato sulla parabola
}

\section{A peer education experience in the high school focused on the parabola}

\author{
Michele Canducci \\ Dipartimento formazione e apprendimento - SUPSI di Locarno, Svizzera
}

Sunto / L'articolo presenta l'esperienza di un primo approccio alla peer education, sia per il docente sia per gli studenti, realizzato in una classe di III liceo. La metodologia utilizzata è quella della peer tutoring, caratterizzata dal lavoro in gruppi composti da studenti tutor di livello disciplinare più alto e da studenti tutorandi di livello disciplinare più basso. Le giornate del percorso sono commentate sulla base dell'analisi di registrazioni effettuate durante i lavori; inoltre si analizzano qualitativamente i questionari e la discussione collettiva realizzate a fine percorso, al fine di individuare i punti di forza e di criticità dell'esperienza. Il focus dell'analisi riguarda principalmente aspetti legati alla metodologia applicata, e non al ruolo giocato dal contesto matematico. Si evidenziano da un lato il carattere di criticità che la peer tutoring riveste laddove non siano mai state realizzate attività cooperative, dall'altro la necessità, per un'efficace azione didattica, di una commistione di approcci didattici sia cooperativi (fra alunni) sia orchestrati dall'insegnante, in un'ottica dialogica che non preveda l'utilizzo esclusivo di un metodo piuttosto che un altro.

Parole chiave: peer education; peer tutoring; apprendimento cooperativo; parabola; orchestrazione.
Abstract / The article presents the experience of a first approach to peer education, both for the teacher and for the students, realized in a III class of high school. The methodology used is that of peer tutoring, characterized by the work in groups made up of students with higher disciplinary level (tutor) and by lower level disciplinary student (tutees). The days of the experience are commented on the basis of the analysis of recordings made during the works; in addition, the questionnaires and the collective discussion carried out at the end of the course are qualitatively analyzed, in order to identify the strengths and weaknesses of the experience. The focus of the analysis mainly concerns aspects related to the applied methodology, and not to the role played by the mathematical context. On the one hand, we highlight the critical nature that peer tutoring plays when no cooperative activities have ever been carried out, and on the other hand, it is pointed out the need, for an effective educational action, to combine teaching approaches both cooperative (among students) and orchestrated from the teacher, in a dialogic perspective that does not foresee the exclusive use of one method rather than another.

Keywords: peer education; peer tutoring; cooperative learning; parabola; orchestration.

\section{Premessa}

II percorso oggetto di questa esperienza e della relazione finale relativa al tirocinio di pratica per l'abilitazione all'insegnamento secondario in Italia, ${ }^{1}$ anno scolastico 2014/2015, si è inserito all'interno di un progetto di dottorato la cui ricerca inten-

1. TFA II ciclo, abilitazione all'insegnamento della matematica e della fisica nelle scuole secondarie di secondo grado in Italia, studenti di età compresa tra i 14 e i 18 anni. 
deva indagare gli effetti di un approccio basato sulla peer education sui processi di insegnamento/apprendimento della matematica. In particolare, in questo articolo viene descritto un percorso che è stato successivamente modificato e rielaborato per essere sperimentato su più vasta scala (Spagnuolo, 2017a) e che qui viene analizzato dal punto di vista degli studenti e dell'insegnante/tirocinante. II contributo ha dunque un carattere improntato più alla riflessione sull'esperienza, piuttosto che alla ricerca di criteri oggettivi di valutazione di efficacia. Inoltre, essendo il primo percorso realizzato in quest'ottica sia da parte del docente che da parte degli alunni, si presentano alcuni aspetti di criticità che sono emersi durante lo svolgimento del progetto e che non erano stati previsti a priori. Attraverso il resoconto delle attività dei gruppi, delle percezioni dell'insegnante e degli studenti, delle criticità emerse, degli aggiustamenti in itinere e delle discussioni collettive, questo articolo intende fornire un contributo alla riflessione sull'efficacia della peer tutoring laddove venga integrata con modalità di orchestrazione da parte dell'insegnante.

Dal punto di vista disciplinare, il percorso affronta l'introduzione della parabola nel piano cartesiano e lo studio del segno di una funzione che ha come grafico una parabola (d'ora in poi indicato con l'espressione: "studio del segno di una parabola"), in relazione alla risoluzione di disequazioni di Il grado. In questo articolo si è scelto di non approfondire l'analisi del ruolo giocato dal contesto matematico, focalizzando piuttosto l'attenzione sulle percezioni degli studenti rispetto alla nuova metodologia utilizzata in classe.

II percorso è stato realizzato nella classe III di un liceo delle Scienze Umane di Rimini, ${ }^{2}$ nell'arco di due mesi, per un totale di 6 interventi, più una lezione introduttiva e una conclusiva.

\section{Quadro teorico: la peer education}

Secondo Comoglio (1996), una prima definizione di peer education può essere data nel seguente modo: «un insieme di tecniche di conduzione della classe nelle quali gli studenti lavorano in piccoli gruppi per attività di apprendimento e ricevono valutazioni in base ai risultati conseguiti».

La ricerca in didattica (Damon \& Phelps, 1989) ha da tempo individuato come, all'interno della dicitura di peer education, si possano ritrovare almeno tre interpretazioni, caratterizzate tutte da un approccio di lavoro tra pari, ma diversificate in alcune caratteristiche strutturali: il peer tutoring, il cooperative learning e la peer collaboration:

«La modalità di peer tutoring presume l'assegnazione dei ruoli di tutori e di tutorandi, laddove si intende generalmente che i primi siano studenti aventi una maggiore padronanza della materia rispetto ai secondi (...). II cooperative learning racchiude tutte quelle forme di peer education caratterizzate generalmente dalla presenza di studenti eterogenei per competenze, in cui non è esplicitamente riconosciuto uno status differente tra i membri di un gruppo. L'organizzazione del lavoro può prevedere la suddivisione dei compiti tra i vari

2. II Liceo delle Scienze Umane italiano è paragonabile al Liceo ticinese con opzione economia e diritto. Gli alunni a cui si fa riferimento nel testo corrispondono, dal punto di vista dell'età, ad alunni ticinesi di classe seconda. 
studenti o l'obbligo di occuparsi tutti insieme di una specifica attività.

Nella peer collaboration i gruppi sono formati in maniera tale che gli studenti abbiano circa gli stessi livelli di competenza e, a differenza del cooperative learning, essi sono chiamati a lavorare in ogni momento congiuntamente sullo stesso problema piuttosto che singolarmente su componenti separate. Nella sua forma originale questa modalità prevede che gli studenti lavorino insieme per risolvere compiti di apprendimento stimolanti che difficilmente riuscirebbero a risolvere da soli».

(Spagnuolo, 2017b, p. 129)

Recentemente, alcuni risultati di ricerca hanno messo in luce come questi metodi di insegnamento/apprendimento si mostrino non sempre efficaci, se vissuti ingenuamente dall'insegnante come panacea per tutti i problemi legati all'apprendimento. A tale proposito, Pellerey (2014) ha messo in luce quanto, se poco guidato e controllato dal docente, lo stesso metodo del cooperative learning risulti fragile. Inoltre afferma che:

"L'insegnamento reciproco tra studenti, il feedback che riceve l'insegnante dagli allievi e quello che egli loro fornisce, la valutazione formativa, I'insegnamento diretto ed esplicito, che segue da vicino la comprensione dei concetti e la padronanza delle abilità, evidenziano una buona validità didattica».

(Pellerey, 2014, p. 262)

In ciascuna delle tre categorie sopracitate il docente assume un ruolo di facilitatore ed organizzatore delle attività, strutturando ambienti di apprendimento in cui gli studenti, favoriti da un clima relazionale positivo, trasformano ogni attività di apprendimento in un processo di problem solving di gruppo, conseguendo obiettivi la cui realizzazione richiede il contributo personale di tutti (Comoglio, 2010).

Tali obiettivi possono essere conseguiti se all'interno dei piccoli gruppi di apprendimento gli studenti sviluppano determinate abilità e competenze sociali, intese come un insieme di abilità interpersonali e di piccolo gruppo indispensabili per sviluppare e mantenere un livello di cooperazione qualitativamente alto. Tali abilità, ovviamente, non nascono spontaneamente, ma deve essere compito del docente trovare strategie adatte a far sviluppare queste competenze (preparare adeguatamente il materiale didattico, supervisionare l'andamento delle discussioni ecc.).

\section{Metodologia}

Dal punto di vista operativo, il percorso si è sviluppato attorno al lavoro degli studenti su schede di attività precedentemente elaborate dall'insegnante e dal dottorando. In questo percorso si è scelto di rielaborare un approccio di peer tutoring con la presenza di ruoli interni ai gruppi: tale approccio è stato pensato in modo specifico per l'insegnamento/apprendimento della matematica ed è descritto in Pesci (2004). La classe di studenti era composta da 18 femmine e 3 maschi, dunque si sono potuti ipotizzare sette gruppi di tre studenti ciascuno. La composizione dei gruppi è stata effettuata tenendo in considerazione sia un'analisi di carattere prettamente disciplinare, fatta sulla base dei risultati di un pretest che mirava a verificare la presenza dei prerequisiti concettuali, sia un'analisi dal punto di vista sociale delle dinamiche 
relazionali fra studenti (descritta dall'insegnante), al fine di raggiungere una suddivisione in gruppi del tipo: un tutor (di livello disciplinare alto) e due tutorandi (di livello disciplinare inferiore).

Poiché l'argomento parabole non era ancora stato affrontato dalla classe, le schede di attività sono state strutturate in ottica di scoperta, in maniera tale che ogni gruppo al suo interno, e poi successivamente durante la discussione di classe, potesse ricostruire e rielaborare autonomamente i concetti trattati. II tutto è stato mediato dall'insegnante/tirocinante e dal dottorando.

Sono state assegnate tre tipologie di ruoli agli studenti, uno per ciascun componente di ogni gruppo, di cui vengono descritti i compiti:

1. Ruolo di helper

- spiegare idee e procedure all'interno del gruppo (l'incaricato espone le varie idee e opinioni);

- chiarire e illustrare (riesporre ciò che gli altri membri hanno detto per spiegare o chiarire un messaggio all'intero gruppo);

- precisare (correggere gli errori nelle spiegazioni degli altri membri, spiegandone il motivo).

2. Ruolo di controller

- controllare i turni (assicurarsi che i membri del gruppo svolgano il compito assegnato secondo i turni prestabiliti);

- incoraggiare la partecipazione (assicurarsi che tutti i componenti del gruppo diano il loro contributo);

- verificare la comprensione (assicurarsi che tutti i membri del gruppo sappiano spiegare chiaramente come si è giunti a una conclusione o una risposta).

3. Ruolo di recorder

- ricapitolare (riassumere ciò che è stato letto e discusso dal gruppo e le conclusioni a cui si è giunti);

- registrare (l'incaricato mette per iscritto le decisioni del gruppo);

- presentare (esporre i risultati raggiunti dal gruppo quando interpellato dall'insegnante)

L'helper è essenzialmente pensato come tutor dal punto di vista disciplinare, il controller e il recorder come tutorandi. Ognuno dei ruoli presenta inoltre dei compiti che corrispondono a funzioni che favoriscono la gestione e l'efficacia interna al gruppo. Inoltre, il recorder aveva anche il compito, quando chiamato dall'insegnante nei momenti di riepilogo e di messa in comune, di esporre quanto raggiunto dal gruppo. La scelta di questi compiti, in parte diversi dalle scelte di ruoli proposti da Pesci (2004), è dipesa da due fattori: in primo luogo, il fatto che si trattasse di una prima esperienza cooperativa della classe ci ha portato a pensare che gruppi con strutture definite fossero maggiormente controllabili, in secondo luogo, poiché si trattava di un tema di matematica nuovo per gli studenti, avevamo bisogno di almeno uno studente per gruppo che potesse trascinare, disciplinarmente parlando, gli altri componenti. Al fine di creare sintonia e dinamiche sociali stabili nel percorso, è stato deciso di mantenere il più possibile i gruppi fissi lungo tutto il percorso, scambiando però al loro interno, dopo ogni attività, i ruoli di controller e di recorder.

Il lavoro all'interno dei gruppi è stato in gran parte registrato tramite telecamera o registratore audio, in modo da poterlo poi visionare/ascoltare e analizzare. 


\section{Aspetti disciplinari e didattici}

\subsection{Considerazioni didattiche}

II rapporto fra geometria e algebra è stato decisamente problematico nella storia della matematica: la conquista del linguaggio simbolico è stata piuttosto lenta e difficoltosa e ci sono voluti diversi secoli per costruire un linguaggio algebrico ed arrivare ad un vero e proprio simbolismo. Le varie quantità, incognite e parametri erano inizialmente indicate con termini del linguaggio naturale, successivamente con abbreviazioni; solo con Viète prima (1540-1603) e Descartes poi (1596-1650) si iniziano ad utilizzare lettere anche per costruzioni geometriche. È chiaro che, riprendendo la distinzione fra la natura degli ostacoli (Brousseau, 1983; D'Amore, Fandiño \& Sbaragli, 2003), siamo davanti ad un possibile ostacolo di tipo epistemologico, cioè un ostacolo all'apprendimento che si riferisce ad un particolare concetto, nodo della matematica, che per sua natura è intrinsecamente complesso e delicato. Non è dunque per nulla scontato e banale per uno studente, proprio perché non lo è stato nel corso dell'evoluzione della matematica, riuscire a gestire i passaggi tra i registri verbale, algebrico e geometrico nell'interpretazione dei parametri che entrano in gioco nell'acquisizione dei concetti che ruotano attorno alla parabola. D'altra parte, è solo grazie alla comprensione e all'utilizzo dei diversi registri semiotici dello stesso oggetto matematico che è possibile concepirlo da diversi punti di vista e costruirsene un modello adeguato (Duval, 2006). $\mathrm{Nel}$ caso di questo percorso si è scelto di insistere molto sul passaggio dal registro geometrico del grafico della parabola a quello algebrico di equazioni e disequazioni di II grado, ipotizzando che molte delle difficoltà concettuali avrebbero infatti riguardato proprio questi aspetti.

Si è scelto di introdurre la parabola come grafico rappresentante l'andamento dello spazio di frenata in funzione della velocità di una vettura, ovvero come modellizzazione di un fenomeno reale. Successivamente ci si è concentrati sulle caratteristiche geometriche dell'oggetto parabola a partire dall'equazione del tipo $y=a \cdot x^{2}+b \cdot x+c$. Si sono tralasciate altre rappresentazioni di equazioni come $y-y_{v}=a \cdot\left(x-x_{v}\right)^{2}$ poiché l'equazione $y=a \cdot x^{2}+b \cdot x+c$ è quella che maggiormente permette di cogliere lo stretto legame tra la parabola e le equazioni e disequazioni di II grado, e anche perché gli studenti non avevano familiarità con la traslazione in geometria analitica. Queste decisioni sono state dettate anche da alcune considerazioni legate al contesto. Trovandoci in una classe di un liceo ad indirizzo umanistico, si è pensato di non approfondire troppo gli aspetti tecnici e algebrici, ma di fornirne una visione globale che permettesse agli studenti di saper leggere, riconoscere e interpretare, nel caso di luoghi geometrici che rappresentano il grafico di una funzione, rapporti di senso fra la coppia di valori $(x, y)$ del piano e la coppia di valori $(x, f(x))$ deducibili dall'equazione del luogo geometrico. Dal tipo di equazione studiata si evince che si è trattato solo il caso di parabole con asse di simmetria parallelo all'asse delle $y$.

Non è stata inoltre trattata la parabola come luogo geometrico, né come intersezione conica. Dal punto di vista algebrico, si è scelto di non fornire la formula dell'ordinata del vertice $y_{v}=-\frac{\Delta}{4 a}$ ma ricavarla per sostituzione di $x_{v}$ all'interno dell'equazione della parabola e di non trattare le formule per ricavare il fuoco e la direttrice.

Per quanto concerne i dispositivi operativi, si è scelto di utilizzare una certa varietà di strumenti di natura e significati diversi: libro di testo, schede per attività di gruppo e compiti a casa, LIM (Lavagna Interattiva Multimediale), software GeoGebra e posta elettronica. 
Tra questi, il vantaggio dell'utilizzo di GeoGebra sta nel fatto di poter fornire con facilità diverse rappresentazioni di un particolare oggetto matematico, favorendo la comprensione e l'interiorizzazione delle relazioni fra le diverse variabili in gioco. L'utilizzo integrato di questo software e della LIM permette di visualizzare, modificare, intervenire graficamente sui vari disegni in modo efficace, salvare il lavoro condiviso in formato digitale, inviarlo tramite posta elettronica ecc.

Vi è un'ulteriore considerazione da effettuare, che riguarda l'idea di matematica e di insegnamento/apprendimento della matematica che hanno gli studenti: spesso chi sceglie, al termine della scuola media, il liceo delle scienze umane lo fa non per un particolare interesse verso le discipline umanistiche e sociali, ma piuttosto per la sicurezza di una poca rappresentanza in termini di ore settimanali di materie scientifiche; il rapporto con la matematica è dunque spesso conflittuale e il rifiuto verso la disciplina assume spesso connotati di ansia e rigetto emotivo (Di Martino, 2015). Ragionando sul progetto e su come poter intervenire in questo senso, si è deciso di inserire nelle varie fasi di realizzazione del percorso spunti di riflessione e discussione riguardanti la matematica, in riferimento a diversi livelli metacognitivi interconnessi fra loro, percepiti dal punto di vista dello studente: I'idea di matematica, I'idea di apprendimento della matematica, l'idea di insegnamento della matematica. In quest'ottica, l'apprendimento fra pari in senso generale ha rappresentato una cornice di senso che ha fatto da cassa di risonanza e amplificato gli aspetti socio-relazionali, disciplinari e metacognitivi dell'apprendimento (Figura 1).

Figura 1

Apprendimento fra pari come cornice di senso e cassa di risonanza.

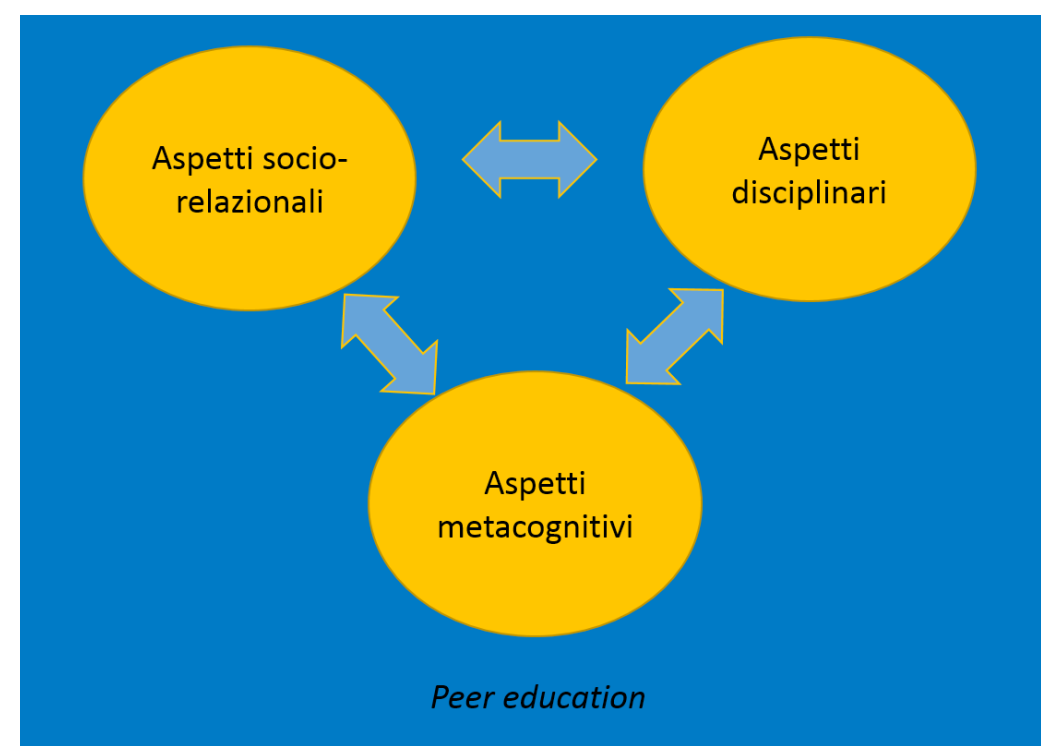

\subsection{Struttura del percorso}

II percorso è stato progettato a priori ed è stato qui schematizzato attraverso una struttura che individua gli snodi concettuali e le relazioni fra le parti:

1. Rappresentazione cartesiana di una situazione fisica in cui emerge un andamento parabolico.

2. Rappresentazione cartesiana di una parabola tramite interpolazione, punto per punto, a partire dall'equazione di una parabola in forma canonica del tipo $y=a \cdot x^{2}$.

3. Caratteristiche geometriche della parabola in forma canonica: I'asse di simme- 
tria, il vertice come punto per cui passa l'asse di simmetria, la concavità e la convessità in funzione del segno del coefficiente $a$.

4. L'equazione generica di una parabola con asse parallelo all'asse delle ordinate $y=a x^{2}+b x+c$.

5. Indagine sul significato geometrico dei coefficienti $a, b$ e $c$.

6. Disegno di una parabola a partire dalla sua equazione: coordinate del vertice, dell'asse di simmetria e ricerca di punti appartenenti al grafico.

7. Interpretazione geometrica di un'equazione di II grado.

8. Segno della parabola: trovare le ascisse $x$ dei punti di un grafico per cui le corrispondenti ordinate $y$ sono positive, negative o nulle.

9. Studio del segno di una parabola a partire dal grafico già dato.

10. Studio del segno di una parabola a partire dall'equazione della parabola: cosa è indispensabile conoscere?

11. Interpretazione grafica di una disequazione di II grado come caso particolare dello studio del segno di una parabola.

Un percorso realizzato sfruttando le metodologie della peer education racchiude inevitabilmente al suo interno anche traguardi di tipo socio-relazionale. Tali traguardi vanno nell'ottica di un'acquisizione di competenze nel saper lavorare in gruppo e riguardano alcune sfere di attitudini/comportamenti fondamentali per una buona riuscita del percorso:

1. saper offrire e ricevere aiuto dall'altro;

2. essere disponibili a dare e ricevere feedback;

3. saper stimolare i membri del gruppo alla partecipazione attiva;

4. saper riconoscere nel gruppo un'opportunità di raggiungimento di obiettivi comuni;

5. saper dare e ricevere fiducia nei confronti degli altri membri del gruppo;

6. sapersi impegnare per interessi condivisi;

7. saper scambiare informazioni e elaborazioni di informazioni e materiali;

8. saper rielaborare un concetto al fine di confrontarlo con gli altri membri del gruppo;

9. saper gestire un clima di serenità che consenta a ciascun membro del gruppo di esprimersi al meglio delle proprie potenzialità.

È importante riconoscere che queste tipologie di obiettivi non sono staccate dai traguardi legati al sapere in gioco; al contrario, gli uni sono di supporto agli altri.

\section{Descrizione dell'intervento}

\subsection{Contesto di sperimentazione}

Nella classe III del liceo delle scienze umane sono dedicate due ore settimanali all'insegnamento della matematica. Salvo piccole variazioni nell'orario, nella fase di svolgimento del progetto si è mantenuta tale impostazione. II percorso si è sviluppato nell'arco di otto settimane.

Sia per gli studenti che per l'insegnante si è trattato della prima esperienza di insegnamento/apprendimento realizzata con metodi cooperativi strutturati tenendo 
conto della letteratura di riferimento. Inoltre, vi è anche da dire che la classe non aveva familiarità con un apprendimento basato sulla scoperta, e che durante l'anno scolastico in corso gli studenti avevano mostrato di essere molto abituati e legati a modalità trasmissive del sapere.

Le conoscenze, abilità e competenze in matematica all'interno della classe avevano una distribuzione piuttosto eterogenea. Questo fatto era dovuto alla presenza di alunni provenienti da percorsi scolastici diversi (tre alunni avevano frequentato il primo anno di liceo scientifico), di alunni con disturbi dell'apprendimento (due alunni con discalculia e uno con dislessia), di alunni non madrelingua da pochi anni in Italia (due alunni immigrati recentemente) e di alunni con frequenza scolastica molto altalenante (due alunni con bisogni educativi speciali). Nonostante ciò, tutti gli allievi della classe avevano una sufficiente familiarità con I'utilizzo di quei registri algebrici e geometrici utili ad affrontare l'argomento parabola e disequazioni di II grado. ${ }^{3}$ Al fine di avere una panoramica dei prerequisiti posseduti dagli studenti, è stato somministrato loro un pretest (Allegato 1). Sulla base dei risultati emersi, è stata formulata una prima ipotesi di gruppi.

Nella lezione successiva di due ore, è stata effettuata in classe una ripresa dialogata e discussa degli esercizi somministrati nel pretest, seguita da un'introduzione sulla metodologia peer education e una spiegazione dei ruoli di helper, recorder e controller. I ragazzi sono in seguito stati suddivisi nei gruppi ipotizzati ed è stato chiesto loro di lavorare su una scheda di esercizi (Allegato 2) attraverso la modalità precedentemente descritta. Questa fase ha permesso al docente e al ricercatore di ottenere, attraverso l'osservazione diretta delle dinamiche sociali che si attivavano, una prima impressione sull'efficacia dei gruppi ipotizzati. Sulla base di queste impressioni, sono stati effettuati, cercando di mantenere un equilibrio tra i vincoli della peer tutoring e le dinamiche relazionali fra alunni, alcuni spostamenti di studenti da un gruppo a un altro e sono stati definiti i gruppi di lavoro, in seguito indicati con una lettera dalla A alla G. Si è cercato il più possibile di mantenere i gruppi fissi, variandone all'interno i ruoli di controller e di recorder ma non il ruolo di helper.

Il percorso ha previsto poi la somministrazione, nell'arco di diversi interventi di lavoro, di sei schede di esercizi e domande aperte, nelle quali si chiedeva di ragionare via via sui nuovi concetti da comprendere e utilizzare. Ogni intervento era costituito da tre fasi principali: un riepilogo dei concetti emersi fino a quel momento; un lavoro a gruppi basato sulle schede durante il quale era garantito il supporto e l'assistenza del docente e del ricercatore; una o più messe in comune, intermedie e finali dalle quali scaturiva l'istituzionalizzazione del sapere in gioco. Come si può notare, in ciascuna delle fasi è in qualche modo presente la figura del docente, o come garante del sapere appreso (prima fase di riepilogo e ultima fase di istituzionalizzazione), o come stimolo e supporto alla comprensione del gruppo (seconda fase). È sembrato importante preventivare questo aspetto al fine di rassicurare gli studenti e arginare fenomeni di spaesamento e disagio nei confronti di un approccio - la peer tutoring in ottica di scoperta - sostanzialmente mai vissuto dalla classe.

In aggiunta alle sei giornate, vi è stato un ulteriore momento conclusivo del percorso che ha visto la somministrazione di un questionario individuale finale a domande aperte, seguito da una discussione collettiva di classe. II questionario è stato pen-

3. Questa classe aveva già affrontato nel percorso di studi un modulo sulle equazioni e disequazioni di primo grado e gli studenti sapevano rappresentare punti e rette nel piano cartesiano. 
sato per raccogliere le percezioni e le opinioni degli studenti riguardo all'esperienza svolta, al fine di individuare snodi problematici su cui riflettere. Le domande del questionario erano:

1. Molti dicono che l'apprendimento collaborativo richiede più impegno ma risulta più efficace: qual è la tua opinione dopo l'esperienza svolta?

2. Come ti sei sentito durante i lavori di gruppo?

3. Cosa ti è piaciuto di più dell'esperienza compiuta? Perché?

4. Cosa ti è piaciuto di meno dell'esperienza compiuta? Perché?

\subsection{Gli interventi del percorso Intervento 1}

Ripresa delle caratteristiche dei ruoli all'interno dei gruppi e suddivisione degli studenti (15 minuti).

Agli studenti, tutti presenti, vengono comunicati i gruppi definitivi di appartenenza, i ruoli iniziali, e dichiarato che, a meno di assenze che avrebbero condotto a cambi durante il percorso, i gruppi sarebbero rimasti fissi. È stato anche comunicato loro che il ruolo di helper sarebbe rimasto fisso in tutto il percorso, mentre i controller e i recorder si sarebbero scambiati i ruoli ad ogni scheda.

Somministrazione prima scheda (Allegato 3): spazio di frenata (1 ora e 30 minuti). La prima scheda di attività conteneva otto esercizi/domande stimolo e mirava a condurre gradualmente gli studenti da una situazione reale modellizzata (spazio di frenata associato al quadrato della velocità) ad un primo studio delle caratteristiche di una parabola in forma canonica, focalizzandone alcune peculiarità grafiche e il significato geometrico del coefficiente $a$. Per consentire un maggior controllo, il lavoro è stato suddiviso a pacchetti di due esercizi per volta: i gruppi avevano 10 minuti per concentrarsi su tali esercizi; al termine del tempo a disposizione, l'insegnante, chiamando a esporre i recorder, ha riepilogato i risultati raggiunti a tutta la classe riportando sulla LIM quanto emergeva dagli studenti. Tale modalità è stata mantenuta durante tutta la lezione, al termine della quale è stato effettuato un riepilogo finale di tutta I'attività. L'ottavo esercizio è stato assegnato come compito per casa da risolvere entro la lezione successiva. Dei sette gruppi formati, quattro sono riusciti a lavorare in modo completamente autonomo $(A, B, C, F)$, tre gruppi hanno invece richiesto spesso l'aiuto del docente ( $D, E, G)$. Emergono delle problematiche dal punto di vista dei ruoli: I'helper rischia di essere l'unico a condurre l'attività, come se fosse il solo a dover svolgere il compito, tanto che nel gruppo $\mathrm{F}$ si sostituisce al recorder nella trascrizione dei risultati raggiunti sul foglio; in due casi (gruppi F e G) il controller si è limitato a gestire i tempi, partecipando poco alla discussione e intervenendo solo per avvisare i compagni sui minuti che mancavano; negli stessi due gruppi il recorder ha semplicemente riportato sotto dettatura quanto gli veniva detto dall'helper. In tre casi $(A, B, C)$ I'helper ha svolto un ruolo positivo ai fini della comprensione dei compagni, attraverso espressioni quali "Avete capito?", oppure "Prova a rispiegarmi".

\section{Intervento 2}

Ripresa della lezione precedente e suddivisione nei gruppi (15 minuti).

La seconda giornata è iniziata con una ripresa dei concetti affrontati la volta prece- 
dente: I'interazione fra docente e studenti ha permesso di riportare l'attenzione sul significato del coefficiente $a$ e di correggere l'esercizio assegnato per casa. L'assenza di due studenti ha costretto a formare un gruppo di quattro persone, composto da un recorder, un helper e due controller. Nello specifico, due componenti del gruppo F hanno lavorato con i due componenti presenti del gruppo E. Prima di iniziare la seconda attività, all'interno dei gruppi è avvenuto lo scambio dei ruoli fra i recorder e i controller. Gli helper sono rimasti come stabilito invariati.

Somministrazione seconda scheda (Allegato 4): i coefficienti a, b e c (1 ora e 30 minuti). L'obiettivo disciplinare della giornata era arrivare ad una graduale scoperta del significato geometrico dei coefficienti $a, b c$ di una parabola con asse di simmetria parallelo all'asse delle ordinate. Dall'analisi dei video è emerso che nei gruppi A e B, come nell'intervento precedente, vi è stata una discussione nella quale l'helper è riuscito a motivare e coinvolgere gli altri componenti del gruppo, stimolando il recorder a scrivere le conclusioni sul foglio e ponendo domande volte a valutare la comprensione di tutti. Nei gruppi $D$ e $E$ I'helper non è riuscito a condurre i lavori e i gruppi si sono appoggiati in modo consistente al supporto dell'insegnante. È sembrato che la presenza di un helper in difficoltà abbia stimolato la presa in carico del lavoro da parte di tutti i membri del gruppo; tuttavia, laddove non si riesce insieme ad arrivare a risultati convincenti in poco tempo, diventa forte il bisogno e la dipendenza dall'insegnante per dipanare dubbi e togliersi da una sorta di "stallo" operativo. Negli ultimi due gruppi ( $\mathrm{C}$ e G) i ruoli di controller e di recorder sono apparsi marginali rispetto a quello di helper, che ha diretto i lavori in modo molto autonomo, senza che ci fosse condivisione o chiarimenti di esercizi o concetti non compresi riferiti all'attività in via di svolgimento. Durante l'attività si è intervallato diverse volte il lavoro interno ai gruppi con momenti di messa in comune orchestrata dall'insegnante con il contributo di tutta la classe. Questo aspetto, seppur presente anche nella giornata precedente, ha avuto in questo caso un'accezione leggermente diversa: sono stati gli studenti a richiedere che alcune considerazioni venissero riprese tutti insieme tramite un momento di messa in comune dei risultati. È risultato inoltre particolarmente importante ai fini della comprensione globale utilizzare GeoGebra, ${ }^{4}$ grazie al quale gli studenti hanno potuto riconoscere, al variare di un parametro per volta, le caratteristiche geometriche dei parametri $a, b$ e $c$. Al termine della lezione si è assegnato un breve esercizio da risolvere entro la lezione successiva (Figura 2).

In base al grafico della parabola disegnata, di equazione $y=a \cdot x^{2}+b \cdot x+c$, completa le scritture inserendo il simbolo opportuno ( '<' minore, '>' massimo, '=' uguale ):
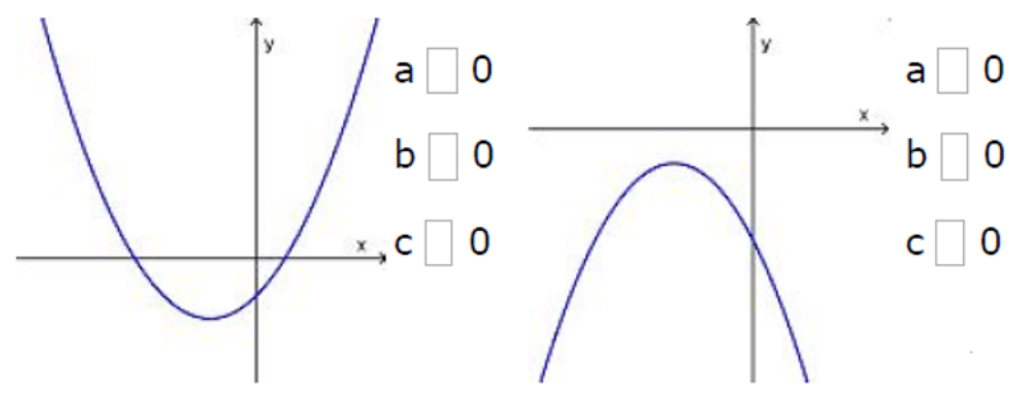

4. I'animazione presentata è disponibile all'indirizzo https://www.geogebra.org/m/ua4GgcaF 
Figura 2

Esercizi assegnati per casa sul significato geometrico dei parametri $a, b, c$.
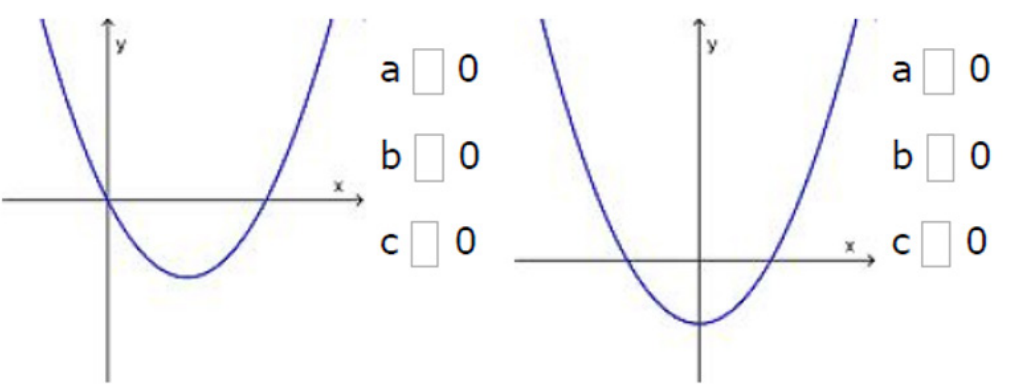

\section{Intervento 3}

Ripresa della lezione precedente e suddivisione nei gruppi (15 minuti)

La terza giornata ha previsto inizialmente la correzione a grande gruppo, gestita dall'insegnante in modalità di discussione collettiva, dell'esercizio assegnato la volta precedente, in modo da riprendere i concetti e aggiornare gli assenti della lezione precedente. In questa giornata erano assenti quattro studenti, tra i quali un helper. È dunque stato necessario ripensare alla composizione interna in modo da variare il meno possibile i gruppi che sembravano più efficienti e a proprio agio. I due componenti dei gruppi $\mathrm{F}$ e $\mathrm{G}$ sono stati uniti rispettivamente ai due componenti dei gruppi $\mathrm{E}$ e $\mathrm{B}$, che sono dunque diventati gruppi composti da quattro alunni (il gruppo $\mathrm{B}$ con un helper e due controller, il gruppo E con due helper)

Somministrazione terza scheda (Allegato 5): equazioni di secondo grado associate alla parabola e coordinate del vertice (1 ora e 30 minuti).

Da un punto di vista disciplinare, I'attività ha consentito di riprendere il ruolo fondamentale del rapporto fra registro geometrico e registro algebrico, rappresentato dalla relazione fra equazione di II grado e intersezione della parabola con l'asse delle $x$. Questo risultato ha permesso anche di introdurre la formula per il calcolo della coordinata $x$ del vertice di una parabola, ${ }^{5}$ I'equazione dell'asse e più in generale una procedura per riuscire a disegnare il grafico di una parabola attraverso le informazioni del vertice, delle intersezioni con gli assi e dell'analisi dei segni di $a, b$ e $c$. A differenza di quanto fatto nelle precedenti due giornate, durante questa lezione c'è stato tempo, nella seconda parte dell'attività, per un lavoro individuale degli studenti nel quale è stato richiesto loro di disegnare in modo autonomo il grafico di una parabola a partire dalla sua equazione.

Nel lavorare sulla prima parte della scheda è emerso a livello globale di classe una problematica dovuta ad una leggerezza commessa in fase di progettazione: dal punto di vista concettuale, per giungere a conclusioni soddisfacenti era necessario far leva su alcuni prerequisiti che, seppur trattati durante l'anno precedente, non erano stati verificati dal docente all'inizio del percorso; inoltre, anche possedendo tali prerequisiti, ci si è resi conto che la richiesta, seppur semistrutturata, ha comportato agli studenti una fatica non preventivata nel ricavare considerazioni e risultati matematici. Queste difficoltà hanno portato a reazioni diverse, emerse durante l'attività e confermate dall'analisi delle registrazioni effettuata a posteriori: in due dei tre gruppi non modificati dai cambi (A e C) si sono attivate interessanti dinamiche di dialogo

5. La formula è stata giustificata attraverso la ricerca della coordinata $x$ del punto medio fra i due punti di intersezione di una parabola con $\Delta$ positivo. 
ed esplorazione nelle quali l'helper non fungeva più da unico garante, ma tutti e tre i componenti si implicavano in prove e tentativi fino ad arrivare in modo autonomo alla soluzione; nei gruppi $\mathrm{B}, \mathrm{D}$ ed $\mathrm{E}$ tale processo è risultato più faticoso e macchinoso, vincolato al sostegno e agli stimoli dell'insegnante.

Come al solito, una volta terminata la prima parte della scheda, l'insegnante ha attivato una messa in comune attraverso la richiesta, fatta ai cinque recorder, di condividere i risultati raggiunti.

I due problemi successivi, relativi al grafico di una parabola a partire dalla sua equazione, sono stati affrontati prima individualmente, poi è stata condivisa la risoluzione all'interno di ogni gruppo; infine, l'insegnante ha chiamato nuovamente a esporre i recorder di ogni gruppo e ha riportato alla lavagna quanto emergeva dalla messa in comune. Nel riepilogo finale, grazie al software GeoGebra integrato con l'utilizzo del software autore della LIM, I'insegnante ha mostrato come la costruzione punto per punto del grafico di una parabola coincidesse quasi esattamente con il grafico che fornisce GeoGebra a partire dall'inserimento dell'equazione della parabola specifica. Ciò ha avuto un effetto rassicurante per gli studenti, tanto che alcuni di loro hanno voluto riprovarlo per altre parabole.

A fine lezione, alcuni studenti hanno espresso la necessità di avere a casa materiali su cui poter studiare. Si è convenuto di inviare di volta in volta agli studenti tramite e-mail le schede di attività realizzate in classe, risolte e commentate. Questa esigenza è nata probabilmente a causa di un senso di spaesamento nei confronti di un apprendimento per scoperta a cui gli studenti non sono abituati. Avere dei materiali a casa, una sorta di appunti della lezione svolta in classe, viene vissuto come aspetto rassicurante.

\section{Intervento 4}

Ripresa della lezione precedente e suddivisione nei gruppi (15 minuti)

L'insegnante ha dapprima condotto una discussione collettiva per riepilogare i risultati raggiunti fino a quel momento, per poi suddividere gli studenti nei gruppi. Poiché era assente solo uno studente che aveva il ruolo di recorder (gruppo F), si è convenuto di lasciare il gruppo formato da due persone piuttosto che distribuirle in due terzetti già formati.

Somministrazione quarta scheda (Allegato 6): il segno di una parabola (45 minuti). Dal punto di vista disciplinare, la scheda proposta aveva l'obiettivo di introdurre e ragionare sul rapporto fra il variare del segno dell'ordinata in funzione dell'ascissa, sia dal punto di vista grafico sia algebrico.

Dall'analisi delle registrazioni, sembra che la maggior parte dei gruppi abbiano lavorato in modo abbastanza fluido. Solo in due casi sono emerse situazioni problematiche che sembrano essere legate in un caso all'interazione sociale fra i membri dei gruppi, nell'altro ad una sovrastima da parte del docente delle competenze matematiche dell'helper: nel gruppo $\mathrm{G}$ il controller e il recorder tacciono e lasciano gestire tutta la scheda all'helper che, da canto suo, non prova a coinvolgere i compagni; nel gruppo $\mathrm{B}$ il controller in difficoltà prova a chiedere aiuto all'helper che dichiara di non essere in grado di spiegare quello che sta facendo e si rivolge all'insegnante.

Anche in questo intervento, è sembrato importante per la comprensione di tutti intervallare momenti di interazione a piccoli gruppi con momenti di discussione del gruppo 
classe orchestrati dall'insegnante. La presenza continua di questa dialettica fra l'interazione studenti-studenti e l'interazione studenti-insegnante sembra avere un effetto confortante sugli studenti: sanno che se anche non riusciranno all'interno del proprio gruppo a raggiungere i risultati richiesti, potranno poi attraverso un momento collettivo colmare gli aspetti non compresi e rielaborare positivamente le difficoltà vissute.

\section{Intervento 5}

Ripresa della lezione precedente e suddivisione nei gruppi (15 minuti)

Nel momento iniziale si è ripreso collettivamente lo studio del segno di una parabola passo per passo con GeoGebra. Gli studenti hanno mostrato di aver apprezzato I'invio tramite e-mail delle schede commentate e risolte, ma hanno anche espresso la mancanza di una visione globale del percorso che permettesse loro di collegare, matematicamente parlando, tutti i concetti in un'unica visione complessiva. Il docente ha rassicurato la classe dichiarando che ci sarebbe stato un momento finale che avrebbe risposto a questa necessità. Poiché non vi erano assenti, i gruppi sono stati formati seguendo la suddivisione originale.

Somministrazione quinta scheda (Allegato 7): disequazioni di II grado (1 ora e 30 minuti).

Girando fra i banchi, ci si è resi conto che alcuni alunni disperdevano energie nella ricerca dei vertici o dell'equazione dell'asse della parabola associata alla disequazione di II grado. Si è più volte interrotto il lavoro e cercato di far comprendere, sempre attraverso una discussione partecipata, come la richiesta che soggiace alla disequazione non implica necessariamente lo studio completo del grafico di una parabola. I tempi si sono inevitabilmente dilatati e non si è riusciti a terminare la scheda di attività, a beneficio però di un feedback verbale positivo da parte degli studenti sulla comprensione del procedimento per risolvere graficamente disequazioni di Il grado. Dall'analisi dei video sono emerse alcune dinamiche interessanti:

1. Come accaduto in altri interventi, nei gruppi F e G, I'helper lavora in modo individuale senza coinvolgere nel processo i due compagni, ma focalizzandosi solo sul compilare la scheda correttamente.

2. Nei gruppi $A$ e $C$, che dall'inizio del percorso non presentano particolari problematiche, è l'helper che riesce a capire come procedere per risolvere l'attività, ma ciò avviene solo grazie agli stimoli e alle domande dei compagni che interagiscono e richiedono spiegazioni puntuali e precise che costringono I'helper a elaborare ipotesi e tentare strategie.

3. Nel gruppo B i ruoli risultano molto mischiati fra loro: si sono create dinamiche di discussione libera sugli esercizi, gli studenti si pongono domande I'uno all'altro a cui provano a rispondere, si correggono e traggono insieme conclusioni; la percezione dall'esterno è che siano tutti insieme i risolutori e che non emerga il ruolo di helper rispetto a quello di controller e di recorder.

4. I gruppi D ed E continuano a faticare dal punto di vista concettuale: risulta necessario un supporto continuo dell'insegnante per poter procedere nella scheda.

\section{Intervento 6}

Somministrazione sesta scheda (Allegato 8): attività conclusiva (1 ora). 
In questa giornata non sono state effettuate registrazioni per evitare che la telecamera provocasse ansia e pressione nella conduzione del lavoro.

Questa attività ha previsto la costruzione del grafico di tre parabole e la risoluzione di tre disequazioni di II grado da parte di ciascun gruppo. In particolare, ad ogni componente del gruppo è stata assegnata la costruzione del grafico di una parabola e la risoluzione di una disequazione di Il grado; I'indicazione del docente era di terminare questa prima parte di lavoro autonomo in 20 minuti.

Allo scadere di questi, i membri di ogni gruppo dovevano condividere tra loro i fogli e, sotto la guida del controller, leggere e discutere il procedimento e le soluzioni trovate da ogni membro, al fine di validarle. In caso di domande, chiarimenti, correzioni, da parte di un qualsiasi membro (quindi anche da parte del controller stesso), il controller doveva fermare la lettura e coordinare la discussione al fine di raggiungere una posizione comune. Una volta raggiunta una posizione comune, era compito del recorder scrivere le eventuali correzioni o aggiunte su ciascun foglio e la risposta finale condivisa dall'intero gruppo.

Degno di nota il caso del gruppo $\mathrm{F}$ i cui componenti erano stati modificati tre volte nel corso del percorso: i ragazzi non sono riusciti a consegnare tutti i lavori e hanno espresso un disagio nei confronti del fatto che, avendo lavorato poco assieme, si erano trovati in difficoltà nel capirsi. Da questo episodio pare emerga una considerazione, e cioè che il linguaggio che si crea in classe debba essere condiviso sia tra il docente e gli alunni ma anche tra gli alunni stessi; sembra infatti che, soprattutto nel lavorare con queste modalità caratterizzate fortemente da aspetti socio-relazionali, I'accettazione reciproca di fattori comunicativi e linguistici sia strutturante rispetto alla qualità del lavoro che si vuol condurre.

\subsection{Questionario finale}

Alcuni studenti avevano espresso l'esigenza di riassumere globalmente i nodi concettuali che si erano via via affrontati durante gli interventi. Per rispondere a questo bisogno è stato scelto, sia per non allungare le tempistiche ipotizzate in fase di progettazione, sia per non forzare ulteriormente le abitudini degli studenti e rassicurarli con una fase conclusiva a loro familiare, di non far costruire agli alunni tale riassunto; è stato dunque l'insegnante, sempre in modalità dialogata con la classe, a presentare una mappa riassuntiva nella quale emergevano gli snodi concettuali degli argomenti trattati (Allegato 9).

È stato quindi somministrato loro il questionario conclusivo del percorso, le cui risposte sono state raccolte e analizzate di seguito. In classe erano presenti 19 studenti su 21. Si è voluto infine procedere ad una discussione collettiva, mediata dall'insegnante, nella quale gli studenti potessero esprimere liberamente la propria idea riguardo al percorso.

Domanda 1. Molti dicono che l'apprendimento collaborativo richiede più impegno ma risulta più efficace: qual è la tua opinione dopo l'esperienza svolta? La Tabella 1 mostra una panoramica delle categorie di risposta degli studenti alla domanda 1. 
Tabella 1

Categorie di risposta degli studenti alla domanda 1.

\begin{tabular}{|l|l|}
\hline CATEGORIE DI RISPOSTA & NUMERO DI STUDENTI \\
\hline a. Più impegnativo e non efficace & 2 \\
\hline $\begin{array}{l}\text { b. Impegno ed efficacia sono uguali } \\
\text { alle lezioni tradizionali }\end{array}$ & 2 \\
$\begin{array}{l}\text { c. Più impegnativo, I'efficacia dipende } \\
\text { dal gruppo }\end{array}$ & 5 \\
\hline $\begin{array}{l}\text { d. Più impegnativo ma efficace o } \\
\text { abbastanza efficace. }\end{array}$ & 2 \\
\hline $\begin{array}{l}\text { e. Non è detto che sia più impegnativo, } \\
\text { ma sicuramente più efficace. }\end{array}$ & 8 \\
\hline
\end{tabular}

Più della metà dei presenti (10 studenti) ha un'opinione complessiva positiva, in termini di efficacia, del percorso tra pari effettuato (categorie d. ed e.). Il protocollo seguente mostra una delle risposte interpretabili in questo senso.

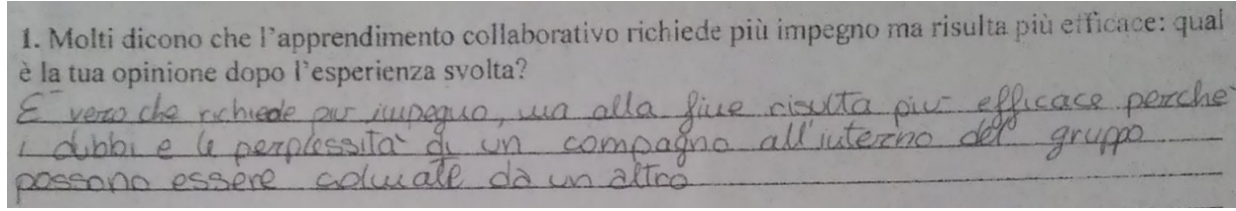

4 studenti, invece, esemplificati dal protocollo seguente, dichiarano di non aver percepito tale tipo di lavoro come utile, o comunque non riconoscono un valore aggiunto di questo metodo per il proprio apprendimento rispetto alla lezione frontale (categorie a. e b.).

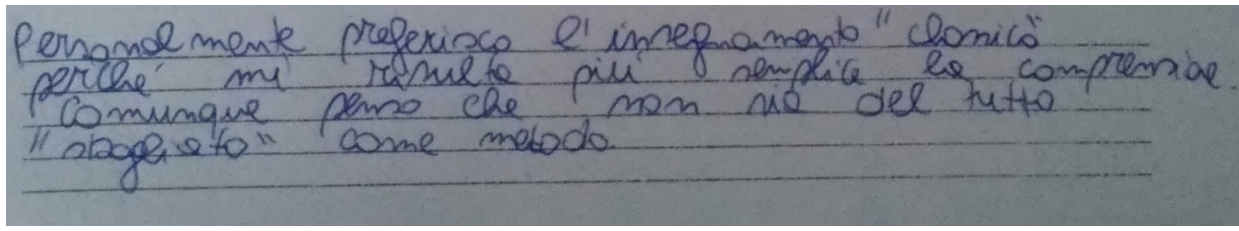

5 risposte, esemplificate dai due protocolli sotto, riguardano gli studenti che dichiarano (categoria c.) come l'efficacia generale non possa essere valutata a prescindere dal gruppo a cui si appartiene o dal tipo di attività e argomenti trattati.

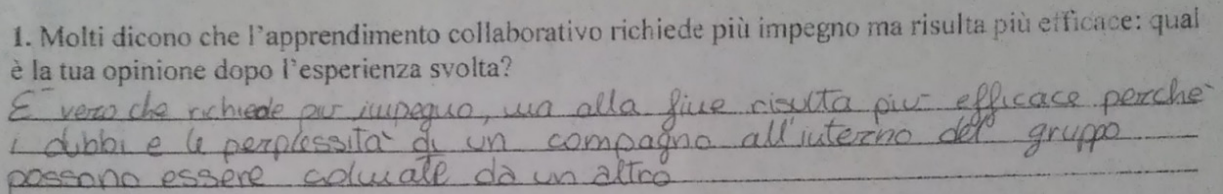


Tabella 2

Categorie di risposta degli studenti alla domanda 2
Molti dicono che l'apprendimento collaborativo richiede più impegno ma risulta piz̀ êficace: qual è fatua oninione dopo l'esperienza svolta?

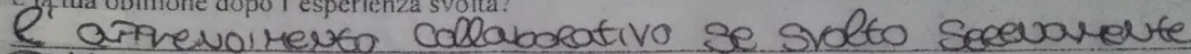

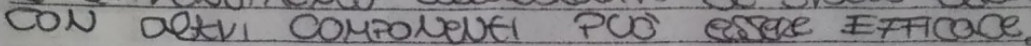

Domanda 2. Come ti sei sentito durante i lavori di gruppo?

\begin{tabular}{|l|l|}
\hline CATEGORIE DI RISPOSTA & NUMERO DI STUDENTI \\
\hline a. Ansia / preoccupazione / sotto pressione & 5 \\
\hline $\begin{array}{l}\text { b. A volte in difficoltà ma abbastanza a } \\
\text { proprio agio }\end{array}$ & 4 \\
\hline c. Bene / a proprio agio / gratificato & 7 \\
\hline d. Incoraggiato / coinvolto & 3 \\
\hline
\end{tabular}

La Tabella 2 mostra le categorie di risposta degli studenti alla seconda domanda. Dall'analisi globale delle risposte emerge la presenza di emozioni sia spiacevoli, associate a stati di ansia o preoccupazione, sia emozioni piacevoli. Nello specifico, su 19 studenti, 9 dichiarano di aver provato in alcuni o molti casi emozioni spiacevoli (categorie a. e b.). Tra questi, i protocolli sotto mostrano come tali emozioni siano da ricercare nel tipo di approccio metodologico proposto e nella percezione delle caratteristiche del ruolo di helper.
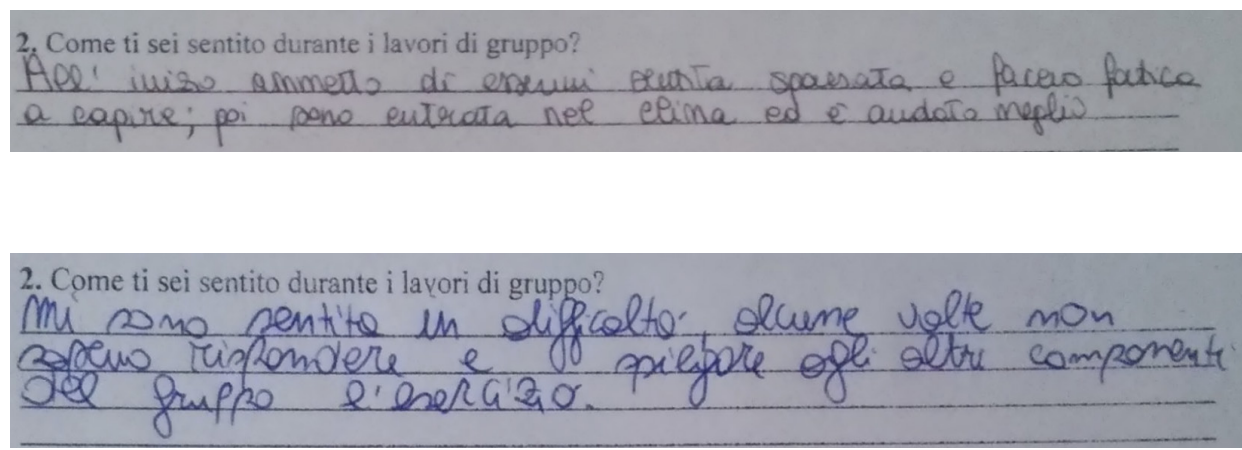

10 studenti invece (categorie c. e d.) dichiarano di essersi sentiti prevalentemente a proprio agio, se non addirittura incoraggiati dal gruppo, come evidenziato dai seguenti protocolli. Nel secondo protocollo si evidenzia anche il caso di uno studente che ha vissuto un'esperienza positiva in termine della propria relazione con la matematica. 
Come ti sei sentito durante i lavori di gruppo?

Duante i laoui di grupeo mi san Butica obbastalta bene perche' c'e'

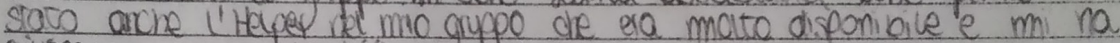
aivtato motro con le difficeltei one auevo.

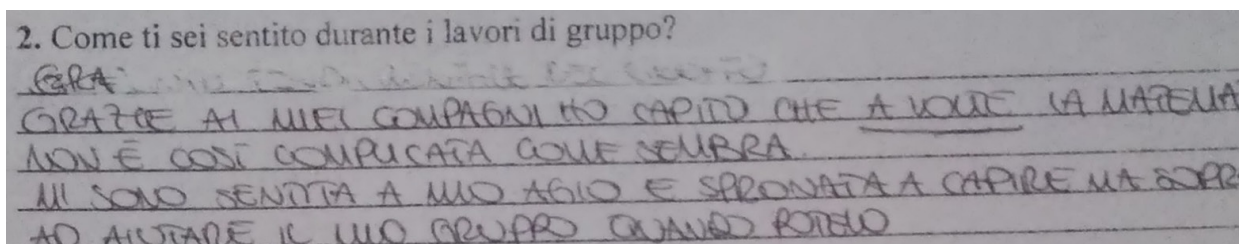

2. Come ti sei sentito durante i lavori di gruppo?

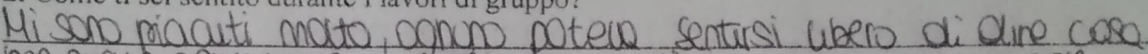

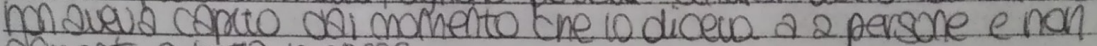
doente a tuta lacosse

Domanda 3. Cosa ti è piaciuto di più dell'esperienza compiuta? Perché?

Tabella 3

Categorie di risposte

degli studenti alla

domanda 3

\begin{tabular}{|l|c|}
\hline CATEGORIE DI RISPOSTA & NUMERO DI STUDENTI \\
\hline $\begin{array}{l}\text { a. Capire da soli / passare dalla pratica } \\
\text { alla teoria }\end{array}$ & 3 \\
\hline $\begin{array}{l}\text { b. Collaborazione / discussione / dialogo / } \\
\text { confronto con i compagni }\end{array}$ & 12 \\
\hline $\begin{array}{l}\text { c. Lavorare insieme ai compagni ma con il } \\
\text { supporto del docente }\end{array}$ & 2 \\
\hline d. Riassunto finale del percorso & 1 \\
\hline e. Sesta giornata: lavoro individuale e poi \\
a gruppo
\end{tabular}

La Tabella 3 mostra le categorie di risposta degli studenti alla terza domanda. II dato che emerge maggiormente riguarda gli aspetti positivi legati al lavorare, collaborare e confrontarsi insieme ad altri compagni. 12 studenti su 19 affermano che la possibilità di discutere e dialogare tra pari sia stato il punto più piacevole di tutto il percorso. Se si considera anche chi vede come fondamentale il supporto del docente al lavoro di gruppo, il numero sale a 14 . Sotto vengono mostrati due protocolli significativi in questo senso; nel secondo viene evidenziato anche il carattere meno monotono e noioso dell'esperienza condotta rispetto alla lezione tradizionale.

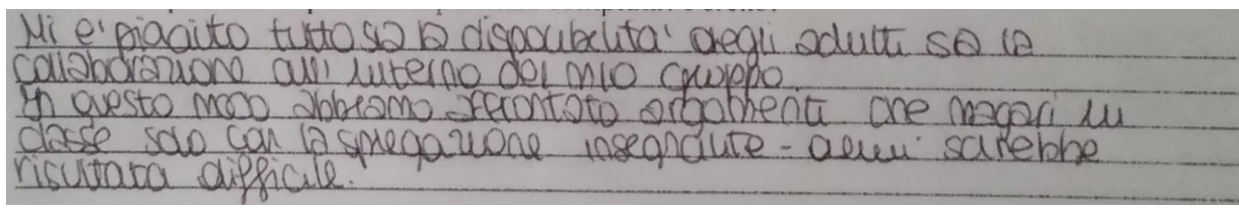




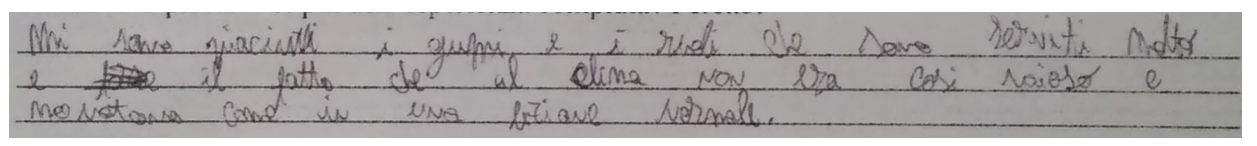

Significativo anche il fatto, esemplificato dal protocollo seguente, che 3 studenti apprezzino il tipo di lavoro cognitivo richiesto, nel quale non veniva proposta una regola da applicare ma si veniva guidati in un ragionamento per arrivare a scoprire significati, costruendo pian piano un'impalcatura di concetti collegati fra loro.

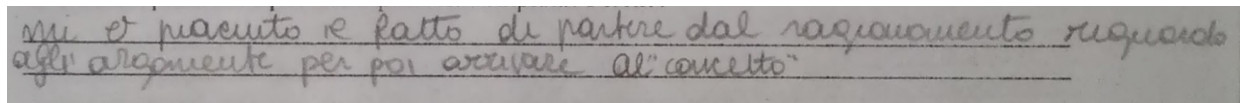

Uno studente ribadisce l'importanza che per lui ha rivestito il momento finale di riepilogo di tutto il percorso appena vissuto attraverso la mappa, mentre un altro sottolinea come sia stato particolarmente significativo il sesto intervento, nel quale è stata messa in pratica una dinamica del tipo lavoro individuale - confronto di gruppo (protocollo di seguito).

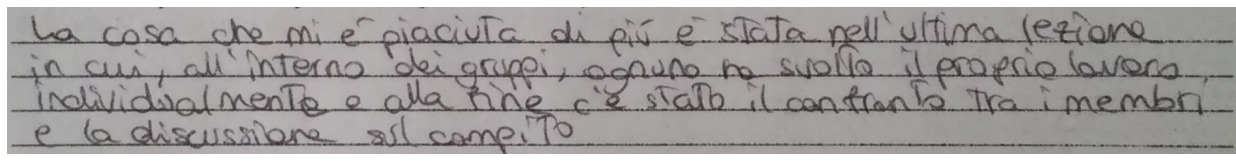

Domanda 4. Cosa ti è piaciuto di meno dell'esperienza compiuta? Perché?

Tabella 4

Categorie di risposta degli studenti alla domanda 4.

\begin{tabular}{|l|c|}
\hline CATEGORIE DI RISPOSTA & NUMERO DI STUDENTI \\
\hline $\begin{array}{l}\text { a. Gestione interna ai gruppi / gestione } \\
\text { dei ruoli }\end{array}$ & 4 \\
\hline b. Apprendimento per scoperta & 11 \\
\hline $\begin{array}{l}\text { c. Mancanza di materiale su cui studiare } \\
\text { a casa }\end{array}$ & 3 \\
\hline d. Niente & 2 \\
\hline
\end{tabular}

In questa ultima tabella (nella quale compaiono 20 risposte in quanto qualche studente ha evidenziato più elementi) emergono le percezioni negative degli alunni rispetto all'esperienza condotta. In primo luogo, come esemplificato dai protocolli sotto, si può notare come l'apprendimento per scoperta sia stato vissuto in modo non facile dalla maggior parte degli studenti (categoria b.). 11 allievi dichiarano infatti di non aver apprezzato il tentativo di condurli ad appropriarsi dell'argomento proposto senza la preliminare spiegazione dell'insegnante. 

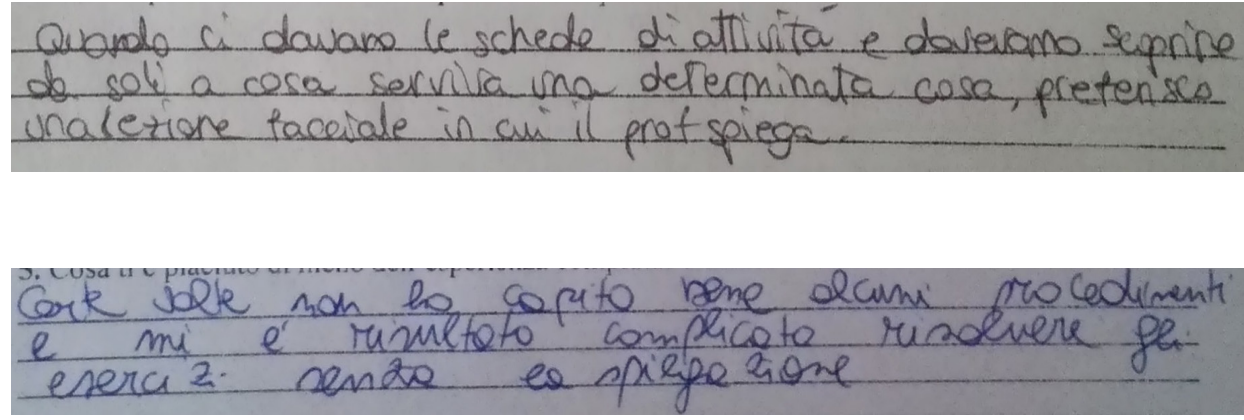

I protocolli di seguito sono riferiti ai 4 studenti (categoria a.) che lamentano di aver vissuto in modo negativo la gestione dei gruppi, sia per quanto riguarda il senso dei tre ruoli, sia per il fatto di aver dovuto modificare spesso i componenti del proprio gruppo (l'autore del protocollo faceva parte del gruppo $F$, modificato tre volte nel corso degli interventi).
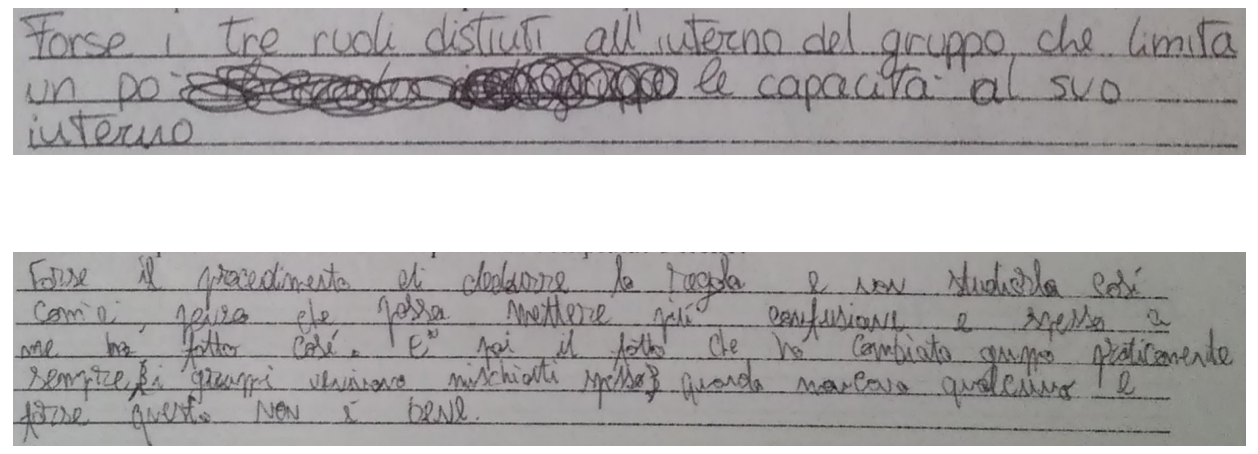

3 studenti (categoria c.) scrivono che la mancanza di materiali su cui studiare e ripassare a casa è stata inizialmente vissuta in modo problematico. Tali studenti, come espresso nel protocollo sotto, evidenziano anche come il dialogo con gli insegnanti sia stato efficace per arrivare a risolvere la situazione.

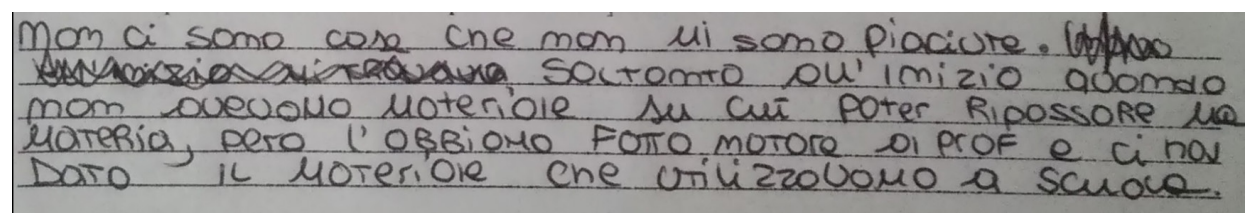

2 studenti infine (categoria d.) dichiarano come non ci sia stato nulla di spiacevole nell'esperienza, e come il percorso sia stato stimolante.

E'stato tuttc beleo e sfimelente 


\subsection{Discussione collettiva}

Terminata la raccolta dei questionari, è stata stimolata dall'insegnante una messa in comune delle considerazioni, dei punti di forza e delle problematiche avvertite dagli studenti. Alcuni alunni hanno scelto di condividere con il resto della classe le proprie idee sull'attività. C'è chi, come F., ha dichiarato di aver «capito molto in questa lezione di riepilogo con la mappa», chi come I. ha sostenuto che

«con questo progetto ogni persona è riuscita a capire come riesce ad apprendere meglio (...) se ha bisogno di fare esercizi insieme a qualcuno o se ha più bisogno di una lezione frontale».

Un'altra studentessa ha confessato di aver avuto la sensazione di «non padroneggiare durante il percorso le conoscenze».

A. invece parla in questi termini:

«lo prof all'inizio ero molto spaesata, non riuscivo proprio perché sono sempre stata abituata a questo metodo della... lezione frontale... quindi ero... però dopo secondo me è stata più una questione di aprirsi... mettersi alla prova... cioè se uno parte già con l'idea "non lo accetto" dopo è difficile che... dopo io sono riuscita».

In seguito alla domanda dell'insegnante: «Lo rifareste?», si è sviluppata una discussione interessante, riportata di seguito.

F. «lo vorrei provarlo con fisica».

M.: «Esatto!».

I.: «lo non so se lo rifarei perché mi sono sentita molta responsabilità e in una materia del genere... insomma...».

A.: «Ad esempio prof io nell'ultima attività... io non sono riuscita a concentrarmi bene sul secondo perché magari ho fatto il primo però... loro avevano bisogno, quindi dovevo aiutare loro e quindi non ho potuto fare il mio meglio». Insegnante: «E per te è stato un male?».

A.: «No, cioè... perché comunque dai se loro hanno capito...».

C.: «Vuol dire che l'ho capito anche io se lo so spiegare... infatti secondo me il problema è quando sei l'helper e non hai capito».

I.: "A me la cosa che gli helper rimanevano fissi non mi è piaciuta per niente. Secondo me anche l'helper doveva cambiare perché alla fine fare l'helper ti mette molto di più alla prova rispetto agli altri due ruoli».

M.: «Ma chi ha difficoltà non può fare l'helper... come fa se non ha capito niente ad aiutare gli altri?».

I.: «Forse potrebbe essere così, che l'helper almeno nelle attività iniziali deve essere uno che ha più facilità, poi potrebbe variare perché a quel punto dovrebbero essere conoscenze abbastanza condivise».

Diverse voci: «Sì, sì esatto».

I.: «Sì però, nel mio gruppo... il fatto che io fossi I'helper non era rilevante perché alla fine ci aiutavamo tutte e bene o male... se magari qualcuno non aveva capito ok, arriva l'helper e cerca di spiegare... però da noi c'era collaborazione e alla fine i ruoli erano... irrilevanti».

V.: «Secondo me il ruolo dell'helper è troppo... cioè è difficile che uno alla pari 
riesca a spiegare come un professore. Cioè è troppa responsabilità secondo me perché non sono un professore che ha tutte le conoscenze d'insieme... però io di andare a spiegare ad un'altra persona quando rischio di avere delle lacune io, trasmetterle a lui.».

C.: «lo infatti mi sono sentita male a un certo punto perché... cioè se io non riuscivo a capire... non riusciva a capire nessuno».

M.: «Beh però infatti comunque c'era sempre il prof o Alessandro».

Insegnante: «L'helper in teoria non doveva essere la persona che... già sapeva le cose... era più un ruolo da guida... guidare la discussione all'interno del gruppo, guidare per cercare strategie, poteva anche essere semplicemente quello che diceva a un certo punto "io non ho capito: chiediamo al prof" ... però è vero... è un ruolo problematico».

(..)

I.: «Un'altra cosa è che essendo in gruppo, ci sono le persone che capiscono di più e quelle che capiscono meno... e quelle che capiscono meno molto spesso stanno in silenzio. Quindi ci si trova in difficoltà, nel senso... nella lezione frontale alla fine è il professore che secondo me riesce a capire subito chi ha capito oppure no; mentre comunque tra coetanei è difficile: io ero I'helper e non riuscivo a capire... cioè per me a un certo punto avevano capito tutti e finiva lì. Poi magari arrivava lei o Alessandro e veniva fuori che non tutti avevano capito e quindi forse questa è una pecca. Però poi alla fine nei momenti di riepilogo finale gestiti da lei... ecco lì era produttivo».

M.: «Nel mio gruppo invece era il contrario. lo ero l'helper e cercavo di far spiegare a loro le cose per capire se avevano capito realmente, verificavo se loro avevano capito».

G.: «lo per esempio nei gruppi... cioè con questa modalità, come ha detto Filippo, mi sono trovata bene. Mi è servita come cosa».

$\mathrm{N}$.: «lo alla fine mi sono trovata bene... però... cioè come abbiamo fatto di raccogliere alla fine tutto quello che avevamo imparato... come abbiamo fatto oggi... però ogni volta».

V.: «Anche magari più esercizi da dare a casa da soli per verificare se abbiamo capito quello che abbiamo fatto in classe perché... arrivavo a casa, magari avevo qualche esercizio però...».

Da questi stralci di discussione emergono alcuni punti di riflessione, in parte già evidenziati. In primo luogo, a parlare e ad esprimere disappunto e criticità sono quasi sempre gli helper: questo ruolo è stato dunque vissuto da chi ne ha avuto l'incarico in modo problematico e, sebbene si riconosca che l'insegnante era comunque sempre disponibile, con una pressione nei confronti degli altri studenti che ha caricato di una responsabilità percepita a volte come eccessiva. Inoltre, le voci degli studenti che esprimono soddisfazione per il lavoro riguardano alunni con andamento scolastico in matematica non positivo: il fatto che per questi studenti il lavoro sia stato efficace potrebbe riguardare il sentirsi a proprio agio nel dichiarare ad un proprio pari, invece che all'insegnante, il non aver capito qualcosa e il sentirsi più liberi di condividere dubbi ed eventuali errori nei ragionamenti matematici (Spagnuolo \& Lazzari, 2018). Un altro dato che emerge riguarda nuovamente il rapporto degli studenti con una consolidata esperienza con la lezione frontale: sembra che, nonostante si riconosca la presenza di momenti di riepilogo conclusivi di ogni scheda e il supporto dell'insegnante e del ricercatore durante i lavori di gruppo, l'abitudine ad una modalità più trasmissiva della conoscenza abbia provocato un generale senso di spaesamento. 


\section{6 contures}

In generale, pur con i distinguo evidenziati nell'analisi, è possibile affermare che l'esperienza nella sua globalità è stata apprezzata dagli studenti, pur avendo avuto un carattere di novità per due motivi: il primo è legato all'apprendimento per scoperta, il secondo alla peer tutoring. Complessivamente, si può affermare che la maggior parte delle criticità emerse e dichiarate dagli studenti hanno riguardato l'affrontare per la prima volta, questi due approcci. Per attenuare questo effetto di spaesamento occorre sicuramente riproporre percorsi analoghi, per far sì che gli studenti si abituino ad un tipo di lavoro nel quale la componente attiva e di costruzione dell'apprendimento è demandata sia all'allievo individualmente che in gruppo. Realizzare altri percorsi di questo tipo è di grande utilità anche per l'insegnante: gli permette di correggere errori di progettazione, familiarizzare con le difficoltà che incontreranno gli studenti, costruirsi una serie di strumenti e pratiche efficaci di gestione dei gruppi. Alcune delle problematiche emerse durante il percorso sono già note nella letteratura relativa all'apprendimento a gruppi, e sono principalmente legate a fenomeni di deriva produttivistica nei quali lavora solo lo studente tutor a discapito della comprensione di studenti più deboli, e casi in cui gli studenti più bravi non si riconoscono e rifiutano il nuovo tipo di lavoro (Carletti \& Varani, 2005, p. 185). Tale rifiuto è risultato quasi assente negli studenti più deboli matematicamente; sembra piuttosto che tali alunni siano quelli che hanno ricavato maggiori gratificazioni sia in termini di comprensione degli argomenti trattati, sia in termini di benessere generale: si affievoliscono sensazioni come la noia e l'inadeguatezza nei confronti delle difficoltà nella disciplina a favore di un maggiore coinvolgimento cognitivo nelle attività proposte e di una maggiore motivazione all'interazione sociale finalizzata alla risoluzione di un compito.

Per quanto riguarda l'efficacia dei gruppi in funzione dei ruoli di helper, controller e recorder utilizzati nel percorso, si può affermare in generale che:

- nei gruppi in cui l'helper, eventualmente dopo il supporto dell'insegnante, riesce a gestire il carico cognitivo delle schede e le dinamiche sociali interne risultano funzionanti (A, B e C), il lavoro interno al gruppo sembra essere efficace per tutti i membri del gruppo;

- nei gruppi in cui I'helper, eventualmente dopo il supporto dell'insegnante, riesce a gestire il carico cognitivo delle schede ma le dinamiche sociali interne sono problematiche $(F, G)$, il lavoro interno al gruppo non sembra essere efficace per il controller e per il recorder;

- nei gruppi in cui I'helper non riesce neanche dopo il supporto dell'insegnante a gestire il carico cognitivo e le dinamiche sociali interne risultano funzionanti ( $D$ ed E), la comprensione dei concetti è vincolata ai momenti di riepilogo e messa in comune a grande gruppo.

Nella modalità di lavoro si è cercato di impostare una continua dialettica fra due estremi della relazione fra studente e apprendimento: da un lato si lasciavano lavorare i gruppi in modo autonomo, dall'altro si sono costantemente realizzati momenti di condivisione e istituzionalizzazione e di intervento sul singolo dubbio interno ai gruppi. Questo continuo rimando fra apprendimento autonomo e orchestrazione dell'insegnante sembra essere stato efficace sia per condurre gli allievi a padroneg- 
giare il sapere in gioco, sia per arginare e rassicurare la classe rispetto allo spaesamento nei confronti di un approccio nuovo all'apprendimento. Dal punto di vista dell'insegnante, il percorso realizzato ha permesso di prendere consapevolezza di quanto sia efficace un approccio alla didattica che tenti di integrare, in modo sinergico e funzionale al contesto classe, varie metodologie.

Per un'analisi generale dell'efficacia dei tre metodi di peer education in relazione alle conoscenze matematiche degli alunni, si rimanda a Spagnuolo (2017a). Quello che interessa qui evidenziare riguarda uno dei risultati della ricerca condotta da Spagnuolo: sembra che quando gli argomenti trattati sono completamente sconosciuti dagli studenti, la modalità di raggruppamento più efficace in termini di apprendimento sia quella di peer tutoring, dunque quella utilizzata nel percorso realizzato e descritto in questo articolo. Analoghi risultati sono emersi anche in una ricerca effettuata con classi della scuola elementare (Arrigo, Maurizi \& Minazzi, 2004).

\section{Bibliografia}

Arrigo, G., Maurizi, L., \& Minazzi, T. (2004). Chi spiega impara a mettere i pensieri bene: la comunicazione intenzionale in matematica. La Matematica e la sua Didattica. Bologna: Pitagora.

Arzarello, F., Ferrara, F., \& Robutti, O. (2011). Mathematical modelling with technology: the role of dynamic representations. Teaching Mathematics and its Applications. 31 (1), 2030.

Brousseau, G. (1983). Théorisation de phénomènes d'enseignement des mathématiques. Thèse de Doctorat d'État. Université de Bordeaux I.

Carletti, A., \& Varani, A. (a cura di) (2005). Didattica costruttivista: dalle teorie alla pratica in classe. Edizioni Erickson.

Comoglio, M. (2000). Educare insegnando. Apprendere ad applicare il cooperative learning, Roma: LAS

Comoglio, M. (1996). Verso una definizione del Cooperative Learning. Animazione Sociale, 4.

D'Amore, B., Fandiño Pinilla, M.I., Marazzani, I., \& Sbaragli, S. (2008). La didattica e le difficoltà in matematica, Trento: Erickson.

Damon, W., \& Phelps, E. (1989). Critical distinctions among three approaches to peer education. International Journal of Educational Research, 13(1), 9-19.

Di Martino, P. (2015). I fattori affettivi e il loro ruolo nell'apprendimento della matematica. L'insegnamento della matematica e delle scienze integrate. 38 A-B (3), 343-362.

Duval, R. (2006). A cognitive analysis of problems of comprehension in a learning of mathematics. Educational Studies in Mathematics, 61(1-2), 103-131.

Morin, E. (2015). Insegnare a vivere. Manifesto per cambiare l'educazione. Milano: Raffaello Cortina editore.

Pellerey, M. (2014). Che cosa abbiamo imparato sul piano della progettazione didattica dalle critiche al costruttivismo in ambito pedagogico? Giornale Italiano della Ricerca Educativa, 13(VII), 262. 
Pesci, A. (2004). Insegnare e apprendere cooperando: esperienze e prospettive, L'insegnamento della matematica e delle scienze integrate, 27A-B(6) 637-670.

Pesci, A. (2010). Cooperative Learning and Peer Tutoring to Promote Students' Mathematics Education.

Spagnuolo, A. (2017a). The effects of the equality parameter on mathematics students' performance. A comparative analysis of Peer Education interventions in teaching-learning of linear and quadratic functions. Tesi di dottorato. Università degli Studi di Ferrara.

Spagnuolo, A. (2017b). Difficoltà nell'applicazione di metodologie cooperative per l'insegnamento della Matematica nella scuola secondaria di II grado. Alcune riflessioni sullo sviluppo dei processi argomentativi. Annali online della Didattica e della Formazione Docente, 9(14), 127-145.

Spagnuolo, A., \& Lazzari, E. (2018). Metodologie di peer education in matematica: alcune riflessioni sulle problematiche socio-relazionali. L'insegnamento della matematica e delle scienze integrate, $41 \mathrm{~B}(1), 53-83$.

Autore/Michele Canducci

Dipartimento formazione e apprendimento - SUPSI di Locarno, Svizzera

michele.canducci@supsi.ch 